\title{
Scalable Memory Hierarchies for Embedded Manycore Systems
}

\author{
Sen Ma, Miaoqing Huang, Eugene Cartwright, and David Andrews \\ Department of Computer Science and Computer Engineering \\ University of Arkansas \\ \{senma, mqhuang, eugene, dandrews\}@uark.edu
}

\begin{abstract}
As the size of FPGA devices grows following Moore's law, it becomes possible to put a complete manycore system onto a single FPGA chip. The centralized memory hierarchy on typical embedded systems in which both data and instructions are stored in the off-chip global memory will introduce the bus contention problem as the number of processing cores increases. In this work, we present our exploration into how distributed multi-tiered memory hierarchies can effect the scalability of manycore systems. We use the Xilinx Virtex FPGA devices as the testing platforms and the buses as the interconnect. Several variances of the centralized memory hierarchy and the distributed memory hierarchy are compared by running various benchmarks, including matrix multiplication, IDEA encryption and 3D FFT. The results demonstrate the good scalability of the distributed memory hierarchy for systems up to $32 \mathrm{Mi}-$ croBlaze processors, which is constrained by the FPGA resources on the Virtex-6LX240T device.
\end{abstract}

Keywords: Distributed memory hierarchy, manycore architecture, embedded system.

\section{Introduction}

Current FPGA densities have reached the Million LUT level, allowing a complete multiprocessor system on programmable chip (MPSoPC) to be configured within a single device. While FPGA density still lags CMOS ASIC's, the malleability of the FPGA fabric provides system designers the flexibility in mixing and matching different types of processors and computational components, tailored to the requirements of each individual application. The use of FPGA's as programmable multiprocessor systems on programmable chips instead of point design custom accelerators has been further enabled by the availability of necessary soft IP system components such as standard busses, soft processors with caches, and multi-port memory controllers. As an example, Xilinx's Microblaze soft processor hosts several standard bus interconnections such as the Processor Local Bus (PLB), XCL bus, Local Memory Bus (LMB), and a Multi-Port Memory Controller (MPMC). The MPMC enables the creation of a Symmetric Multiprocessor (SMP) shared memory architecture for up to 7 processors plus 
standard bus interconnection. FPGA fabrication technology continues to follow Moore's law and vendors are promoting next generation devices where the number of processors along with transistors will double every 18 months. This fabrication capability promises future systems with 100's to 1,000's of processors integrated within a single chip. This capability promises significant advancements in scalability, portability, and design reuse. Scalable performance can be achieved through the use of parallel programming models and software design flows instead of more tedious and time consuming custom circuit designs. Design space exploration can occur through re-compilation instead of re-synthesis. Importantly MPSoPC's composed of standard bus and memory hierarchies and programmable processors will bring significant increases in design portability between logic families and across chip generations.

A key aspect of enabling this vision is the ability to support the new class of programming models emerging for manycore chips with scalable numbers of processors. Historically, programming models evolved as abstractions for existing architectures. The asynchronous multithreaded programming model has become popular for shared memory parallel machines. The multithreaded programming model is a suitable abstraction for multiprocessor systems with linear global address spaces, or systems in which all processors have visibility and access to the complete memory system. While models based on linear global address spaces are adequate for SMP architectures, the SMP architectures themselves have known scalability issues. Recently, interest has once again peaked in traditional non-uniform distributed memory hierarchies due to their ability to eliminate the classic SMP bottleneck associated with a global bus and single shared global memory. Unfortunately newly emerging multi-tiered memory hierarchies break the linear global address space assumption upon which many of our historic programming models are based. New programming models such as OpenCL are beginning to emerge as more suitable abstractions for the multi-tiered distributed memory hierarchies anticipated for manycores with 100's to 1,000's of processors. Within this new class of programming models, processors do not have visibility or access to all memory locations. In a significant departure with prior models, the memory hierarchy is no longer hidden under layers of abstractions and instead is now explicitly exposed to the programmer. Program execution times can depend on how well programmers understand and manage marshaling of data between memory tiers and address ranges. Explicit movement of program instructions between memory tiers also unfortunately eliminates the benefits of implicit transfer of instructions between memory and traditional cache organizations that occurred transparently under the global linear address abstractions. Still, the multi-tiered distributed memory hierarchies show promise for scalability and increased processor-memory bandwidth type metrics over much "flatter" SMP systems with caches. The challenge in enabling these next generation programming models for MPSoPC's is to provide necessary run time system services, processing resources, and multi-tiered memory hierarchy structures upon which the models rely. 
Table 1. Characteristics of 5 Memory Hierarchies

\begin{tabular}{|c|c|c|c|c|}
\hline & ry Hierarchies & Instructions & $\overline{\text { Data }}$ & Test Outcomes \\
\hline 1 & \multirow{2}{*}{ SMP } & No cache & \multirow{2}{*}{ No cache } & Base case \\
\hline 2 & & \multirow{2}{*}{ Cached } & & Caching instructions \\
\hline 3 & Hybrid & & & Distributed data access \\
\hline$\frac{4}{5}$ & $\begin{array}{c}\text { Distributed } \\
\text { 2-tiered }\end{array}$ & \begin{tabular}{|l|} 
Distributed (PLB bus) \\
Distributed (LMB bus)
\end{tabular} & & Bus latency \\
\hline
\end{tabular}

In this paper, we present our exploration into how multi-tiered memory hierarchies can effect the scalability of systems with 100's to 1,000's of processors. New programming models such as OpenCL introduce new sets of system service calls layered on top of existing asynchronous multithreaded programming model infrastructure such as pthreads. For our explorations we take the same approach and build additional system service calls on top of our hthreads microkernel [6]. Hthreads was developed as a unifying abstraction within which designers could develop threads that run as custom hardware accelerators and software threads, as well as software threads running across heterogenous mixes of processors within an SMP model. Hthreads provides a full set of pthreads compliant run time system services for thread synchronization, management, and scheduling operations. Hthreads is structured as a hardware/software co-designed microkernel, and has been implemented on Xilinx family FPGA's. A detailed description of hthreads and it's prior uses can be found in 6. For this work, hthreads allows direct comparison of a high level application running on SMP+cache based systems and with additional system service wrappers, distributed multi-tiered memory hierarchies with explicit data marshaling. We provide direct comparisons for systems with up to 6 slave processors, the limit of current MPMC components. We then provide evaluations for the same applications and system services running on different multi-tiered memory hierarchy configurations with up to 32 processors, the limit of our current FPGA components. An important outcome of our work is an analysis of how different configurations of multi-tiered memory hierarchies effect overall application run time performance, and an analysis on the effects of both weak and strong scalability. Our results represent actual run times of the applications and our system services, and not theoretical results predicated with simplifying assumptions.

\section{Memory Hierarchies}

We are interested in the five memory hierarchies listed in Table 1 and shown in Fig. 1. The first two memory hierarches 1,2 represent traditional centralized global memories from our SMP architectures. The last two hierarchies 4,5 are distributed memory hierarchies. Memory hierarchy 3 is a hybrid, with instructions being centralized in the global memory and data being distributed within local memories. All five memory hierarchies are implemented on Xilinx FPGA 


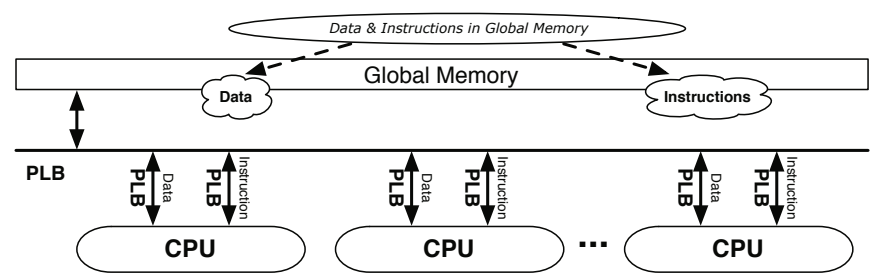

(a) Memory hierarchy 1: shared memory for both data and instructions

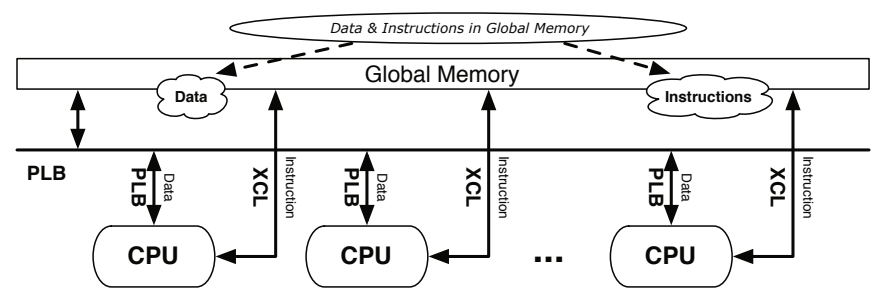

(b) Memory hierarchy 2: shared memory for both data and instructions with i-cache

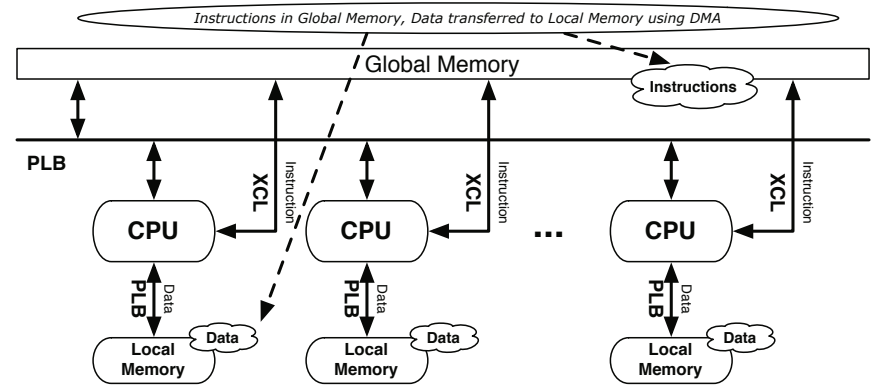

(c) Memory hierarchy 3: distributed memory for data and shared memory for instructions

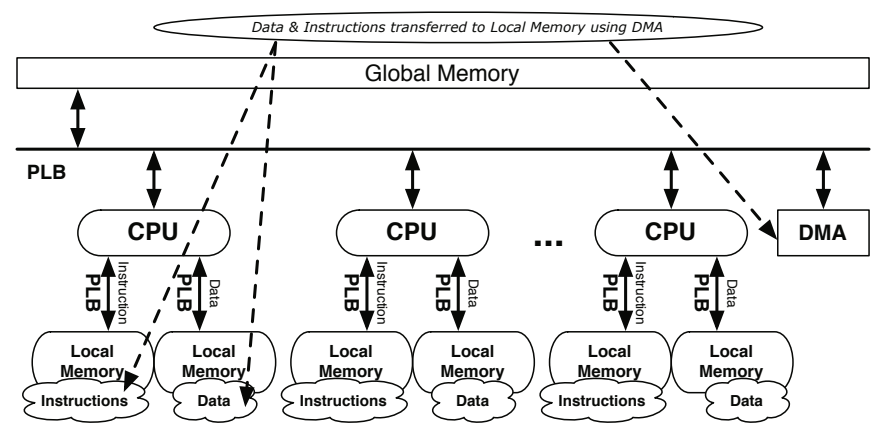

(d) Memory hierarchy 4: distributed memory for both data and instructions with slow local bus

Fig. 1. Various memory hierarchies 


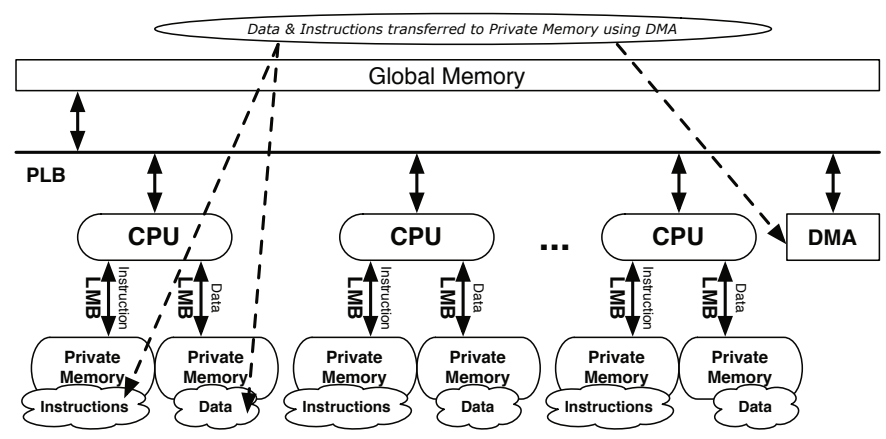

(e) Memory hierarchy 5: distributed memory for both data and instructions with fast local bus.

Fig. 1. (Continued)

ML507 or ML605 boards, with off-chip DRAM used for global main memory. Local memories are implemented using distributed BRAMs. In all cases, data and instructions are originally stored in the main memory. Final results are saved in the main memory as well. In all memory hierarchies the off-chip main memory is connected to the main PLB bus through one MPMC port.

- In Memory Hierarchy 1 (Fig. 1(a) both instruction port and data port of each MicroBlaze processor are connected to the PLB bus.

- In Memory Hierarchy 2 (Fig. 1(b) the instruction port of each Microblaze is connected directly to one MPMC port. Processor data ports are connected to the PLB bus. The instruction cache uses the Xilinx CachLink (XCL).

- In Memory Hierarchy 3 (Fig. 1(c) each instruction port is still connected to the off-chip main memory through an MPMC port. The instruction cache uses XCL. However, the data port is connected to a local memory module (through a local PLB connection). Before processing occurs, data are transferred using DMA from the main memory to the local memory. Final result data are transferred back to the off-chip memory later using DMA.

- In Memory Hierarchy 4 (Fig. 1(d) both instruction port and data port of each MicroBlaze processor are connected to a local memory through separate PLB busses. Before processing occurs, data and instructions are transferred from the off-chip memory to local memory modules using DMA. Final result data are transferred back to the off-chip memory using DMA after the computation finishes.

- Memory Hierarchy 5 (Fig. $1(\mathrm{e})$ is similar to memory hierarchy 4 with the exception that LMB busses replace PLB busses as connections between local memories and MicroBlaze processors.

Note that within the current EDK tools used, caches for each Microblaze require the use of XCL links connected directly to the MPMC port. Therefore, the data caches were turned off in Memory Hierarchies 1,2. 


\section{$3 \quad$ Experiments and Results}

\subsection{Experimental Setup}

All application programs were configured to operate in a master-slave mode. A main program runs on the master processor and distributes work loads to slave, or worker processors. The PowerPC on the ML507 and a MicroBlaze on the ML605 serve as the master processors, respectively. Each master processor has a direct connection to one of the MPMC ports with instruction cache enabled. The data port of the master processor is connected to the main PLB bus. Work loads are distributed to each worker processor as a single thread kernel function. Each thread kernel function is compiled into a thread ELF (Executable and Linkable Format) file. This ELF file is stored in the main memory before execution. The start address and the size of the ELF file are given to the DMA engine that transfers kernel functions into local memory. The master processor is only responsible for creating the worker threads and initiating DMA transfers between the main memory and local memories.

\subsection{Weak Scalability Analysis}

Our first analysis concerns how the memory hierarchy effects the notion of weak scalability. Weak scaling defines how execution time varies when the computational load is linearly increased with the number of processors. In the ideal case, given a work load $\mathcal{W}$ on one processor, the work load for $N$ processors should be $N \mathcal{W}$, which is evenly divided in $N$ processors. We chose matrix multiplication and IDEA (International Data Encryption Algorithm) encryption as representative applications to evaluate weak scalability. We implemented both matrix multiplication and IDEA encryption on a Xilinx ML507 board containing a Virtex-5FX70T device. Matrix multiplication is the representative of applications in which memory is frequently accessed for input data. Conversely, IDEA encryption is a more instruction-intensive application in which a series of eight identical transformations and an output transformation are performed for each 64-bit plaintext input, resulting in the generation of a 64-bit ciphertext. We use both benchmarks to comprehensively test the 5 different memory hierarchies for up to 6 worker threads, the limit of the MPMC for memory hierarchies 2,3. Fig. 2 shows the run time results.

In the matrix multiplication application, each node is given two $20 \times 20$ matrices, $A$ and $B$, in which each element is a 32-bit integer. The computation involves $C=A \times B$ and $D=B \times A$. For distributed memory configurations $A$ and $B$ data arrays are transferred from the main memory to the local memory using DMA. Correspondingly, the $C$ and $D$ arrays are transferred back to the main memory. The volume of each DMA transfer is 3,200 bytes with a latency of approximately $50 \mu \mathrm{s}$. The kernel size of matrix multiplication is 2,520 bytes with a transfer latency of approximately $40 \mu \mathrm{s}$. For IDEA encryption, the size of both the plaintext and ciphertext is 3,200 bytes. The data transfer latencies are the same as in matrix multiplication. The kernel size of IDEA is 3,468 bytes with a DMA transfer latency of approximately $55 \mu \mathrm{s}$. 


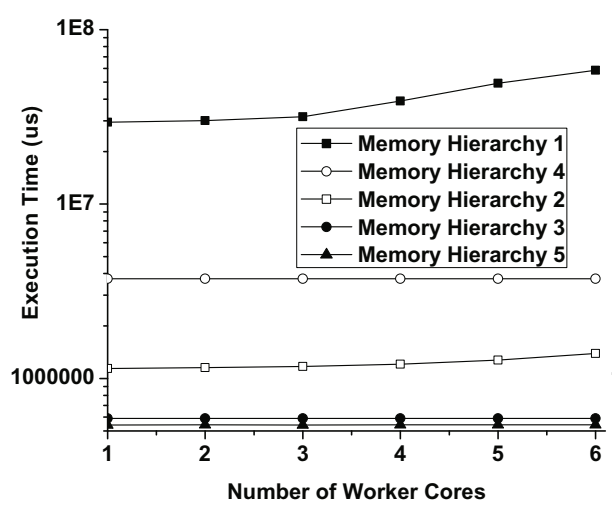

(a) Matrix multiplication

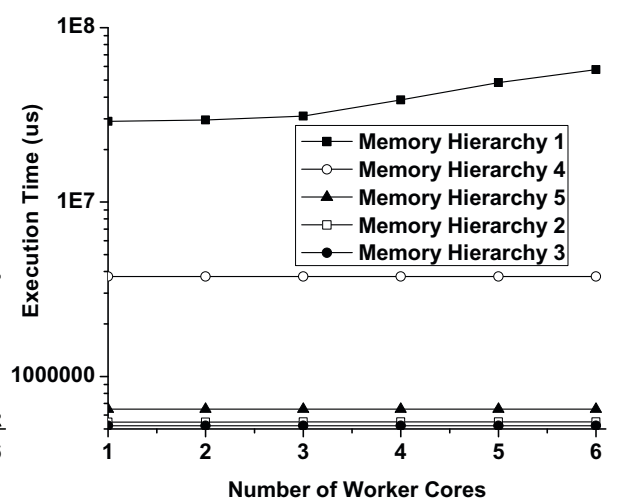

(b) IDEA encryption

Fig. 2. Performance comparison of benchmarks on 5 memory hierarchies (Note: (1) Instruction cache size: $8 \mathrm{~KB}$, local memory size: $8 \mathrm{~KB}$; (2) Matrix multiplication kernel size: 2,520 bytes, IDEA kernel size: 3,468 bytes; (3) DMA transfer time: data in $\rightarrow 50$ $\mu s$, data out $\rightarrow 50 \mu s$, instructions $\rightarrow 40 \mu s$ (matrix multiplication) / $55 \mu s$ (IDEA)).

Memory Hierarchy 1. For scalable systems, the end-to-end computation time should be approximately the same when the number of cores grows. In Fig. 2 , Memory Hierarchy 1 exhibits the worst scalability. This is expected for SMP systems with no data or instruction caches. All memory requests are through a common bus. A system with a single worker core takes 29 seconds to finish the computation for both applications. The computation time then grows nonlinearly as more cores are added into the system. The computation time almost doubles for the 6-core system. Clearly traffic contention on the main PLB bus, and the contention for the centralized memory contributes to the nonscalability of this memory architecture.

Memory Hierarchy 2. In Memory Architecture 2, the instruction cache is enabled and connected to the global memory through the MPMC. A cache size of 8 $\mathrm{KB}$ is sufficient to accommodate all instructions of both applications. Thus only compulsory and conflict cache misses occur but no misses due to capacity. Bus contention on the main PLB bus is now only due to data requests. For both applications, the computation time is dramatically reduced compared with the first architecture. However, the matrix multiplication and IDEA applications demonstrate different performance trends. For matrix multiplication, the computation time continues to grow as the number of cores increases, with the growth at times exhibiting nonlinearity. Matrix multiplication is a data-intensive application in which the computation of one element in the result matrix involves reading one row and one column from the two source matrices. Thus the nonlinearity can be primarily attributed to the increased contention on the main PLB bus. On the other hand, the IDEA is a computation-intensive application. Since there are only 400 64-bit plaintexts, each processor generates 400 memory read and 400 memory write requests each of which is of 8 bytes. This represents a low volume 
Table 2. Performance Comparison of Various Instruction Cache Sizes on Memory Architecture 3 (unit: $\mu s$ )

\begin{tabular}{c||c|c|c|c|c|c}
\hline \multirow{2}{*}{$\begin{array}{c}\text { Number } \\
\text { of Worker }\end{array}$} & \multicolumn{3}{c|}{ Matrix Multiplication } & \multicolumn{3}{c}{ IDEA $^{\star \star}$} \\
\cline { 2 - 7 } Core & Instruction Cache Size (byte) & \multicolumn{2}{c}{ Instruction Cache Size (byte) } \\
\cline { 2 - 7 } & 256 & 1024 & 8192 & 256 & 1024 & 8192 \\
\hline \hline 1 & $1,602,136$ & 589,413 & 589,405 & $2,493,714$ & 825,241 & 520,919 \\
\hline 6 & $2,062,022$ & $1,474,003$ & 590,093 & $3,680,123$ & $1,164,336$ & 521,660 \\
\hline
\end{tabular}

^ Matrix multiplication kernel size: 2,520 bytes.

** IDEA kernel size: 3,468 bytes.

of data transfer and will not result in any significant bus contention. For Memory Hierarchy 2 instructions are stored in the off-chip memory and are transferred into the cache largely due to compulsory misses during startup. The small size of the IDEA program will fit entirely into the cache. With minimal data contention and the use of the instruction cache, the IDEA encryption application demonstrates good scalability on Memory Architecture 2. The combined results show how even moderate contention across a single bus effects scalability.

Memory Hierarchy 3. In Memory Hierarchy 3, each worker core is equipped with an $8 \mathrm{~KB}$ local memory connected across a PLB bus. The instruction port of each core is connected to a cache and global memory through the MPMC. The results shown in Fig. 2 indicate good performance scalability for both applications. The total execution time increases by approximately $100 \mu s$ for each additional core. The $100 \mu s$ increases are contributed to the data transfer latencies between off-chip global memory and the local memory. Although this memory hierarchy is still centralized from the point of view of the instructions, it is distributed during the runtime. The $8 \mathrm{~KB}$ cache is bigger than both application kernels. Therefore, all instruction accesses take place locally once the entire kernel is loaded into the cache. However, in normal cases, the cache is typically smaller than an application so that frequent memory access would be required during runtime. Table 2 shows the performance results for both applications for different cache sizes. The results show how execution time increases when the cache size decreases. This increase is more evident for the computation-intensive IDEA application. For both the matrix multiplication and IDEA encryption applications, Memory Hierarchy 3 loses the scalability when the cache size is reduced below what is necessary to eliminate capacity misses. As an example the execution time for the 6 -core system running IDEA is $50 \%$ more than the 1-core system.

Memory Hierarchies 4,5. Both Memory Hierarchies 4,5 are distributed 2tiered memory architectures in which both data and instructions are DMA'ed to local memories. The only difference between the two hierarchies is the type of local bus used to connect the processor to the local memory. Both the LMB-based and PLB-based architectures exhibit good scalability with the LMB slightly better. The slight increase in execution times for increased numbers of cores 
Table 3. Performance Comparison of Various Options of Local Busses on a 1-core System (unit: $\mu s$ )

\begin{tabular}{c||c|c|c|c}
\hline \multicolumn{1}{c||}{ Application } & \multicolumn{4}{c}{ Data local bus - Instruction local bus } \\
\cline { 2 - 5 } & PLB-PLB & LMB-PLB & PLB-LMB & LMB-LMB \\
\hline \hline Matrix Multiplication & $3,730,825$ & $3,725,673$ & 614,080 & 541,154 \\
\hline IDEA & $3,745,240$ & $3,744,717$ & 653,599 & 648,696 \\
\hline
\end{tabular}

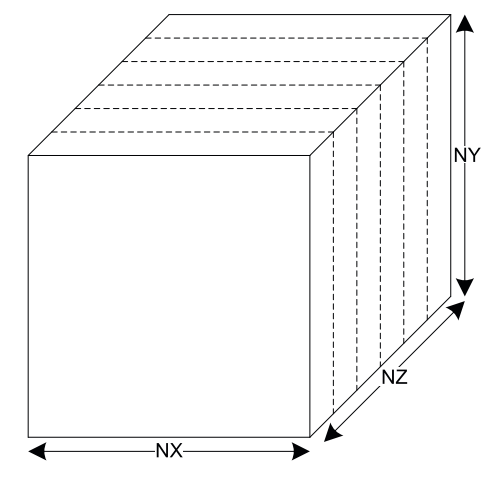

(a) 2D FFT in $\mathrm{X}-\mathrm{Y}$ dimensions.

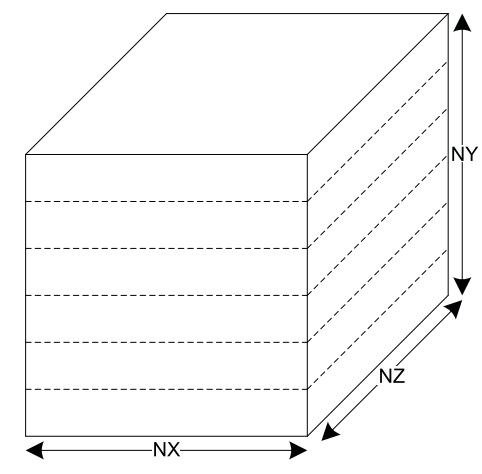

(b) 1D FFT in Z dimension.

Fig. 3. Two steps in $3 \mathrm{D}$ FFT

is mainly attributed to the additional data and instruction transfer latencies. Table 3 shows the timing results for the four different configurations of busses between local memory and the data and instruction ports of the MicroBlaze processor. First, no significant difference is observed between using a PLB or LMB bus configuration to the data port. For both applications, the difference is very small when different data bus is used while the instruction bus is the same. Conversely, the use of LMB in place of a PLB for the instruction bus shows significantly reduced execution times. Overall, Memory Hierarchy 5 demonstrates the best performance and scalability for all 5 hierarchies listed in Table 1. Particularly, the MPMC cannot support a system in which the number of worker nodes is bigger than 6 . Therefore, Memory Hierarchy 5 is adopted in the study of strong scalability in which a $3 \mathrm{D}$ FFT is carried out on system up to 32 cores. Due to the limited resource on Virtex-5FX70T device, the 3D FFT is implemented on a Virtex-6LX240T device on the Xilinx ML605 board.

\subsection{Strong Scalability Analysis}

As shown in Fig. 3, the computation of 3D FFT can be performed in two steps. In the first step, the FFT along X and Y dimensions are carried out. The data are distributed to individual processors along the $\mathrm{Z}$ dimension. Once the computation of the first step finishes, an all-to-all data transpose are carried out to re-distribute the data along the $\mathrm{Y}$ dimension. In a typical case, this all-to-all 
Table 4. Performance Results of 3D FFT on 3 Different Platforms of Memory Hierarchy 5 (unit: $\mu s$ )

\begin{tabular}{|c|c|c|c|c|c|c|c|}
\hline \multirow{3}{*}{$\begin{array}{c}\text { Number } \\
\text { of Worker } \\
\text { Cores }\end{array}$} & \multicolumn{7}{|c|}{ Performance Breakdown in 7 Stages } \\
\hline & DMA_D(ata) & DMA_I(str.) & Kernel & DMA_D & DMA_D & Kernel & $\overline{\mathrm{DMA} D}$ \\
\hline & $\mathrm{D}(\mathrm{ram}) 2 \mathrm{~B}(\mathrm{ram})$ & $\mathrm{D} 2 \mathrm{~B}$ & $2 \mathrm{D}$ & $\mathrm{B} 2 \mathrm{D}$ & $\mathrm{D} 2 \mathrm{~B}$ & $1 \mathrm{D}$ & $\mathrm{B} 2 \mathrm{D}$ \\
\hline$\overline{4}$ & 3,822 & 557 & $1,944,370$ & 4,101 & 3,690 & 771,932 & 4,108 \\
\hline 16 & 4,388 & 1,898 & 486,651 & 4,813 & 4,385 & 178,565 & 4,818 \\
\hline 32 & 4,501 & 3,653 & 247,842 & 4,987 & 4,525 & \begin{tabular}{|l|}
93,848 \\
\end{tabular} & 5,004 \\
\hline & \multicolumn{2}{|c|}{ Total Execution time } & \multicolumn{3}{|c|}{ 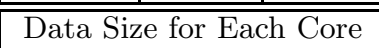 } & \multicolumn{2}{|c|}{ Kernel Size } \\
\hline 4 & \multicolumn{2}{|c|}{$2,987,148$} & \multicolumn{3}{|c|}{65,788 bytes } & \multicolumn{2}{|c|}{8,124 bytes } \\
\hline 16 & \multicolumn{2}{|c|}{963,412} & \multicolumn{3}{|c|}{16,636 bytes } & \multicolumn{2}{|c|}{7,056 bytes } \\
\hline 32 & \multicolumn{2}{|c|}{693,361} & \multicolumn{3}{|c|}{8,444 bytes } & \multicolumn{2}{|c|}{6,836 bytes } \\
\hline
\end{tabular}

* The matrix initialization time and the global data transpose time are not listed in the breakdown, but are counted in the total execution time. Both operations are carried out by the master processor.

transpose should be carried out in all threads in which one thread sends the proper data to other $N-1$ threads and receives the data from them as well. In our case, the all-to-all transpose is carried out by the master node. The whole computation can be divided into the following stages:

(1) The data of X-Y plane(s) are sent to worker processors;

(2) The kernel instructions are sent to worker processors;

(3) The worker processors compute the 2D FFT separately;

(4) The results of 2D FFT are transferred back to the off-chip memory;

(5) The data along the $\mathrm{Y}$ dimension are sent to the worker processors;

(6) The worker processors compute the 1D FFT separately;

(7) The results of 1D FFT are sent back to the main memory.

The same kernel can compute both 2D FFT and 1D FFT. In order to reduce the kernel size, the computation of sin and cos functions is realized using lookup tables.

The size of the 3D matrix is fixed at $32 \times 32 \times 32$. Each element consists of the real part and the imaginary part, each of which is presented in the single precision floating-point precision. Three different manycore systems of Memory Hierarchy 5 are implemented and the performances are summarized in Table 4, As shown in the table, the kernel execution times for both 2D FFT and 1D FFT are linearly reduced as the number of worker cores increases, demonstrating an excellent strong scalability. Since the problem size is fixed in this case, the transfer volume of data stays the same as the number of cores varies. Therefore, the DMA transfer time is almost the same for different manycore systems, although the transfer time slightly increases when the same amount of data is divided into smaller pieces as more cores are added into the system. The only time that linearly grows is the instruction transfer time. The size of the instructions does not change much for different manycore systems. Since the instructions need to be 
sent to each individual worker node, the total time for transferring instructions is directly related to the number of cores.

\section{Related Work}

Most our previous manycore systems designed with hthreads [6] used the Memory Hierarchy 2 if the number of cores was $\leq 6$. For systems more than 6 cores, Memory Hierarchy 1 was adopted, which demonstrated poor scalability. The local memory indeed is the scratchpad memory mentioned in many previous work. In [5, 7, 3, 9] several algorithms were proposed to partition instructions or data into the scratchpad memory for reducing the cache miss rate and the energy consumption. However, most of them assumed a fixed-size cache and scratchpad memory in the memory hierarchy. In [1] a reconfigurable instruction memory hierarchy for embedded system was proposed in which instructions were assigned to scratchpad memory and cache to maximize the performance. Their work was extended in 2. so that the configuration between the scratchpad memory and the cache can be changed during the different phases of an application. However, these previous work focused only on a single-node platform and did not address the scalability of manycore systems. Scalable memory hierarchy has been investigated in the field of general purpose chip multiprocessor (CMP) as well. Most of previous work in this field focused on the distributed cache and highly-efficient interconnect 4. In 8], a cache hierarchy including L1, L2, and the last level cache was studied focusing on the design tradeoff of the last level cache. A twolevel cache architecture was proposed in [10] consisting of private L1/L2 cache and shared L3 cache. The private cache is next to each processing core and only stores the private data. The L3 cache is a central one and saves the data shared by all cores. Our results already show that a distributed cache architecture alone can not provide the scalability for applications as the number of cores grows.

\section{Conclusions}

As the number of cores on embedded systems keeps growing as predicted by Moore's law, the original centralized memory hierarchy needs to be re-designed to provide the performance scalability. In this work, we evaluate the performance scalability of distributed multi-tiered memory hierarchies in which the data and the instructions are transferred to local memory next to the worker processor. Five different memory hierarchies, 2 centralized, 1 hybrid, and 2 distributed, are compared and analyzed on Xilinx FPGA devices. Through a detailed benchmarking using real-life applications such as matrix multiplication, IDEA, and 3D FFT, it is shown that the distributed memory hierarchy, in which the local memory can provide a access latency comparable to the cache, demonstrates an excellent performance scalability for embedded manycore systems up to 32 cores on a Virtex-6LX240T device. 
Currently, the local memory sizes for both data and instructions are fixed and pre-decided. One size may not fit well for all applications. Therefore, one of the future work is to analyze the application source code to figure out the size for local memory accordingly, i.e., the size is application-specific. Other future work include the design of scalable interconnect to provide efficient cross-node communication during runtime.

\section{References}

1. Ge, Z., Lim, H.B., Wong, W.F.: A reconfigurable instruction memory hierarchy for embedded systems. In: Proc. 15th International Conference on Field Programmable Logic and Applications (FPL 2005), pp. 7-12 (August 2005)

2. Ge, Z., Wong, W.F., Lim, H.B.: DRIM: A low power dynamically reconfigurable instruction memory hierarchy for embedded systems. In: Proc. Conference on Design, Automation and Test in Europe (DATE 2007), pp. 1-6 (April 2007)

3. Kandemir, M., Choudhary, A.: Compiler-directed scratch pad memory hierarchy design and management. In: Proc. 39th Annual Design Automation Conference (DAC 2002), pp. 628-633 (June 2002)

4. Kumar, A., Peh, L.S., Kundu, P., Jha, N.K.: Express virtual channels: towards the ideal interconnection fabric. In: Proc. 34th Annual International Symposium on Computer Architecture (ISCA 2007), pp. 150-161 (June 2007)

5. Panda, P.R., Dutt, N.D., Nicolau, A.: On-chip vs. off-chip memory: the data partitioning problem in embedded processor-based systems. ACM Trans. Des. Autom. Electron. Syst. 5(3), 682-704 (2000)

6. Peck, W., Anderson, E., Agron, J., Stevens, J., Baijot, F., Andrews, D.: Hthreads: A computational model for reconfigurable devices. In: Proc. 16th International Conference on Field Programmable Logic and Applications (FPL 2006), pp. 885888 (August 2006)

7. Steinke, S., Wehmeyer, L., Lee, B.S., Marwedel, P.: Assigning program and data objects to scratchpad for energy reduction. In: Proc. Conference on Design, Automation and Test in Europe (DATE 2002), pp. 409-415 (March 2002)

8. Thoziyoor, S., Ahn, J.H., Monchiero, M., Brockman, J.B., Jouppi, N.P.: A comprehensive memory modeling tool and its application to the design and analysis of future memory hierarchies. In: Proc. 35th Annual International Symposium on Computer Architecture (ISCA 2008), pp. 51-62 (June 2008)

9. Verma, M., Wehmeyer, L., Marwedel, P.: Cache-aware scratchpad allocation algorithm. In: Proc. Conference on Design, automation and test in Europe (DATE 2004), pp. 1264-1269 (February 2004)

10. Yan, S., Zhou, X., Gao, Y., Chen, H., Luo, S., Zhang, P., Cherukuri, N., Ronen, R., Saha, B.: Terascale chip multiprocessor memory hierarchy and programming model. In: Proc. 2009 International Conference on High Performance Computing (HiPC 2009), pp. 150-159 (December 2009) 